\title{
Effect of the hard magnetic inclusion on the macroscopic anisotropy of nanocrystalline magnetic materials
}

\author{
I. Navarro, E. Pulido, P. Crespo, and A. Hernando \\ Instituto de Magnetismo Aplicado, RENFE-UCM, Laboratorio Salvador Velayos, P.O. Box I55, \\ Las Rozas, 28230 Madrid, Spain
}

It is shown that the presence of highly anisotropic magnetic precipitates in a soft multiphased matrix can produce a remarkable hardening, even when the volume fraction of the precipitates is small. The exchange coupling between the matrix and the precipitates is the relevant parameter. In particular, the simplified analysis we develop in this paper accounts for the magnetic hardening observed in very soft Fe-rich nanocrystals after annealing at higher temperatures.

\section{INTRODUCTION}

Iron-rich nanocrystals exhibit outstanding magnetic properties as soft magnetic materials. $\mathrm{Fe}-\mathrm{Si}-\mathrm{B}-\mathrm{Cu}-\mathrm{Nb}$ is a good example of soft nanocrystal. ${ }^{1}$ The alloy is cast as an amorphous ribbon. A subsequent heat treatment at $520^{\circ} \mathrm{C}$ gives rise to a drastic decrease of the coercive field from $100 \mathrm{mOe}$ (in as cast state) to $5 \mathrm{mOe}$. X-ray and TEM analyses show that an ultrafine structure of $\mathrm{Fe}-\mathrm{Si}$ nanocrystals is embedded into the amorphous matrix. The grain size of the bec $\mathrm{Fe}_{3}$ Si-type grains is about $10 \mathrm{~nm}$. For such average grain size the exchange correlation length, derived from the local anisotropy constant of the amorphous matrix and the crystalline anisotropy of the bec crystallites, which is close to $8 \times 10^{3} \mathrm{Jm}^{-3}$, is much larger than the grain dimensions. Therefore, the structural macroscopic anisotropy vanishes. ${ }^{2}$ Furthermore, for this critical crystallization step the macroscopic magnetostriction averages out so eliminating any anisotropy originated by residual stresses via magnetoelastic coupling. The lack of both anisotropy and magnetostriction is the cause of the drastic softness of the material reached by nanocrystallization.

After further annealing at higher temperatures a dramatic increase of the coercive field of about four orders of magnitude occurs (from $5 \mathrm{mOe}$ to $25 \mathrm{Oe}$, value reached by annealing at temperatures above $620^{\circ} \mathrm{C}$ ). ${ }^{3}$

As the macroscopic anisotropy of a random anisotropy system increases with the sixth power of the average grain diameter, it was claimed the increment of grain diameter to be the cause of the magnetic hardening. ${ }^{3}$

However, thoroughly performed determinations of grain size have shown that the average diameter of grains grows from 8 to $12 \mathrm{~nm}$ when the annealing temperature increases from $520^{\circ}$ to $620^{\circ} \mathrm{C}$. Therefore the increment of grain size cannot be claimed as origin of such four order of magnitude increment of coercitivity. ${ }^{4}$

Some recent observations performed by Mössbauer spectroscopy, DSC, TEM, and $\mathrm{x}$-ray diffraction techniques have pointed out that the magnetic hardening should be related to the appearance of $\mathrm{Fe}-\mathrm{B}$ precipitates as well as a small volume fraction of paramagnetic phase. ${ }^{4}$ The aim of this report is to show how the anisotropic tetragonal Fe-B crystallites highly diluted in the soft nanocrystalline matrix drastically increase the macroscopic anisotropy.

\section{THEORETICAL BACKGROUND}

A common characteristic of prominent soft, as nanocrystals, and prominent hard, as $\mathrm{Nd}-\mathrm{Fe}-\mathrm{B}$, magnetic materials is the coexistence of different phases. The macroscopic anisotropy depends on both the distribution of the local anisotropy constants and the intensity of the exchange coupling between different grains and phases. ${ }^{5}$ It has recently been shown that exchange coupling between grains can decrease noticeably the coercive field of hard magnets. ${ }^{6,7}$

Let us analyze the effect of the $\mathrm{Fe}-\mathrm{B}$ precipitates on the macroscopic anisotropy of nanocrystals. As the exchange correlation length is larger than $1 \mathrm{~mm}$ the magnetic moments of $\mathrm{Fe}-\mathrm{Si}$, in the absence of $\mathrm{Fe}-\mathrm{B}$ precipitates, do not exhibit any average anisotropy. It is well known that $\mathrm{Fe}-\mathrm{B}$ precipitates appear after the full nanocrystallization of $\mathrm{Fe}-\mathrm{Si}$ as a crystallization of the residual amorphous matrix. Therefore we will consider a few crystallites of $\mathrm{Fe}-\mathrm{B}$ embedded into a structure of nanocrystals of $\mathrm{Fe}-\mathrm{Si}$ which forms the soft matrix. Exchange interactions act between $\mathrm{Fe}-\mathrm{Si}$ grains and between $\mathrm{Fe}-\mathrm{Si}$ and $\mathrm{Fe}-\mathrm{B}$ grains. The effect of a crystallite of $\mathrm{Fe}-\mathrm{B}$ can be envisaged by taking into account the following simple model. Consider a discrete system, consisting of a linear chain of atomic moments (soft phase) coupled by exchange interphase interaction, to atomic magnetic moments with uniaxial anysotropy (hard cluster). The soft magnetic moments are coupled by exchange intraphase interaction. The spatial orientation equilibrium distribution of the magnetic moments in the remanent state and that originated upon application of demagnetizing fields was analytically calculated.

In order to introduce the basic ideas underlying in our study let us consider the switching process at $0 \mathrm{~K}$ of one atomic moment, with uniaxial anisotropy constant $D$ and coupled by exchange interaction $\gamma 2 J S^{2}$ to a set of $L$ identical atomic moments, which interact among themselves with exchange interaction $2 J S^{2}$; see Fig. 1. Considering an applied magnetic field antiparallel to the atomic moments 


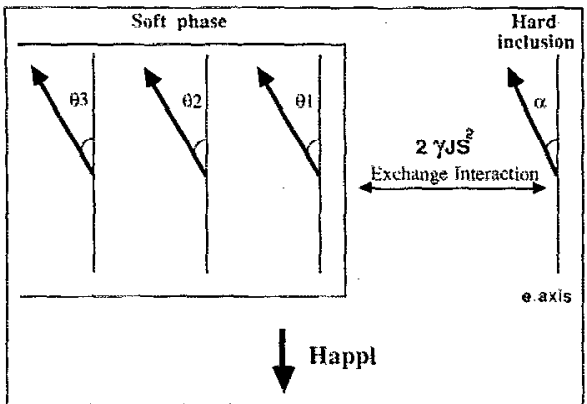

FIG. 1. Simplified diagram of the discrete system analyzed in Eq. (1) (System 1).

we can express the free energy $E_{T}$ of the system as:

$$
\begin{aligned}
E_{T}= & D \sin ^{2} \alpha-\gamma 2 J S^{2} \cos \left(\theta_{L}-\alpha\right) \\
& -\sum_{1}^{L-1} 2 J S^{2} \cos \left(\theta_{i}-\theta_{i+1}\right)-\mu_{0} m H \cos (\pi-\alpha) \\
& -\sum_{1}^{L} \mu_{0} m H \cos \left(\pi-\theta_{i}\right)
\end{aligned}
$$

where $\alpha$ and $\theta_{i}$ are the angles formed between each moment and the easy axis of the hard magnetic moment.

\section{DISCUSSION}

The equilibrium configuration is found minimizing $E_{T}$ in Eq. (1), for a given applied demagnetizing field $H$, with respect to $\alpha$ and $\theta_{i}$. This leads, for $H=0$ and $\gamma=0$ (the $L$ soft moments are exchange decoupled to the hard magnetic moment ), $\alpha=\theta_{i}=0$. When $H$ is increased an infinitesimal value, the energy minimization is obtained for $\alpha=0$ and $\theta_{i}=\pi ;$ only in case $H$ reach the anisotropy field, $\alpha$ becomes $\pi$. If $\gamma \neq 0$, the applied field cannot reverse abruptly the magnetic moments because the interphase exchange coupling forces them to remain parallel to the hard magnetic moment. The result is an increase of the applied magnetic field necessary for switching all the atomic moments: that

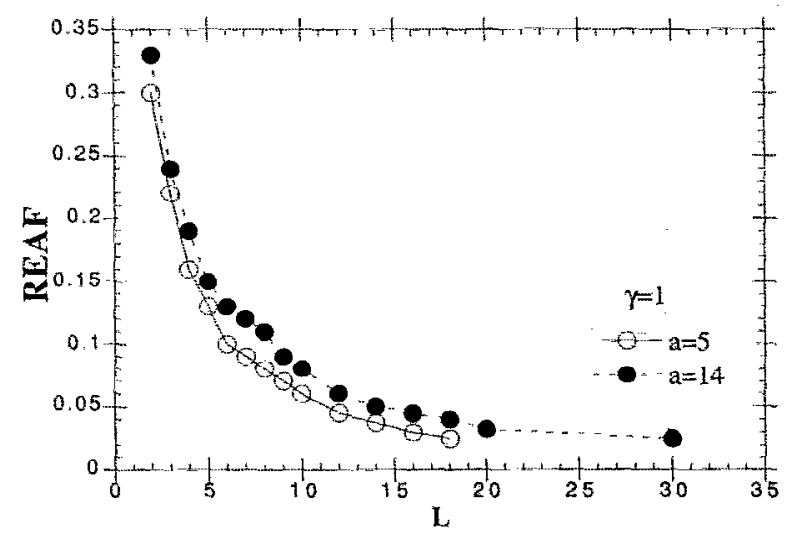

FIG. 2. Dependence of REAF, with the number of soft magnetic moments $L$ for $a=5$ and $a=14$. Results shown correspond to the system 1 and $\gamma=1$.

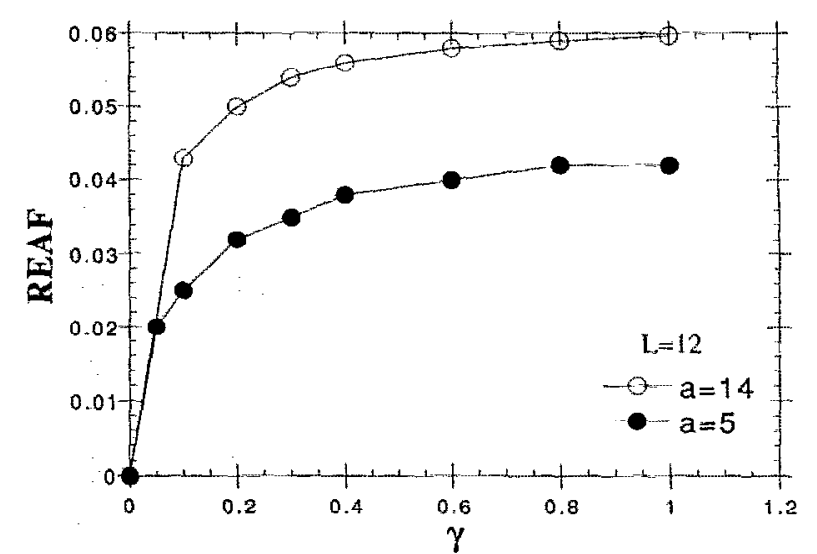

FIG. 3. Dependence of REAF on the parameter for $a=5$ and $a=14$, in the system 1 and for $L=12$.

is to say an increase of the effective anisotropy field of the total system, EAF. We defined the reduced effective anisotropy field, REAF as $\mu_{0} m$ times EAF divided by $2 D$.

In Fig. 2, we plot the reduced effective anisotropy field, REAF, as function of $L$. We consider $\gamma=1$, and value for $a=2 J S^{2} / 2 D$ of $a=14$ which roughly correspond to the ratio for $\mathrm{Fe}_{2} \mathrm{~B}\left(K \sim 5 \times 10^{5} \mathrm{Jm}^{-3}\right.$ and $\left.A_{\mathrm{ex}} \sim 10^{-11} \mathrm{Jm}^{-1}\right)$. As it can be observed the switching field strongly depends on the number of soft magnetic moments $L$. In case of $L=12$ (corresponding to a volume fraction of $\mathrm{Fe}_{2} \mathrm{~B}$, observed by Mössbauer experiments, ${ }^{4}$ about $8 \%$ ) the value of the reduccd anysotropy ficld is roughly $6 \times 10^{-2}$, which corresponds with a switching field about $750 \mathrm{Oe}$. It is interesting to mention that a remarkable magnetic hardening of the soft phase is produced, even when the volume fraction of the precipitate is small.

Let us study the role played by the intraphase and the interphase exchange in the switching process in the system described above. Figure 3 shows the dependence between REAF and $\gamma$, phenomenological coefficient of interphase exchange coupling, for two values $(a=5,14)$ of the ratio of exchange to anisotropy energies. As we can see, the interphase exchange interaction increase the magnetic hardening.

In the following we will analyze in a more realistic configuration with different orientations of easy axes of the precipitate, the efficiency of the interphase exchange in the hardening process. Let us consider the soft phase limited at both extremes by $\mathrm{Fe}_{2} \mathrm{~B}$ precipitates whose easy axes lay perpendicular, see Fig. 4. Applying a magnetic field $H$ pointing antiparallel to one of the hard magnetic moments we can express the total free energy as

$$
\begin{aligned}
E_{T}= & D \sin ^{2} \alpha_{1}+D \sin ^{2} \alpha_{2}-\gamma 2 J S^{2} \cos \left(\theta_{1}-\alpha_{1}\right) \\
& -\gamma 2 J S^{2} \sin \left(\theta_{I}+\alpha_{2}\right)-\sum_{1}^{L-1} 2 J S^{2} \cos \left(\theta_{i}-\theta_{i+1}\right) \\
& -\mu_{0} m H \cos \left(\pi / 2+\alpha_{2}\right)-\mu_{0} m H \cos \left(\pi-\alpha_{1}\right) \\
& -\sum_{1}^{L} \mu_{0} m H \cos \left(\pi-\theta_{i}\right),
\end{aligned}
$$




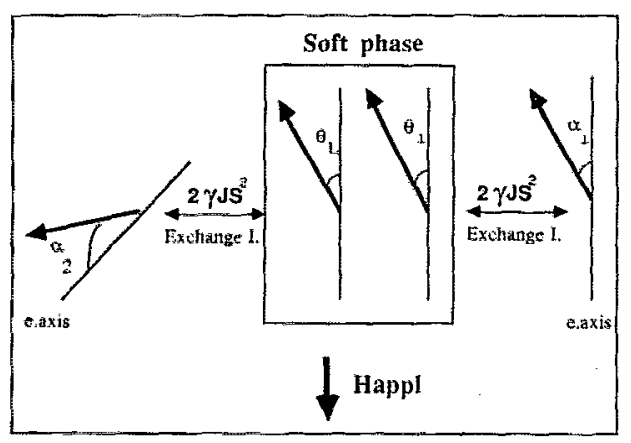

FIG. 4. Simplified scheme of the discrete system analyzed in Eq. (2) (System 2).

where $\alpha_{1}$ and $\alpha_{2}$ are the angles formed by each hard moment and their corresponding easy axis and $\theta_{i}$ is the angle formed by the soft moment and the easy axis parallel to the applied field.

The dependence of REAF on $\gamma$ is presented in Fig. 5 for a value of $a=14$ and $L=20$, the plot reflects that interphase exchange coupling increase the REAF relatively to the decoupled $(\gamma=0)$ system. As we can sce this curve presents a different behavior from that in Fig. 3. The exchange coupling contributes in a different way due to the perpendicularity between easy axes of the hard magnetic inclusions. Even in the worst case, $\mathrm{REAF}=0.014$, that corresponds to a switching field about $175 \mathrm{Oe}$, a considerable magnetic hardening in the global system is produced. The drastic effect of the highly anisotropic precipitates in the magnetic hardening process through the interphase exchange coupling is evidenced in Fig. 6 . We plot the nor-

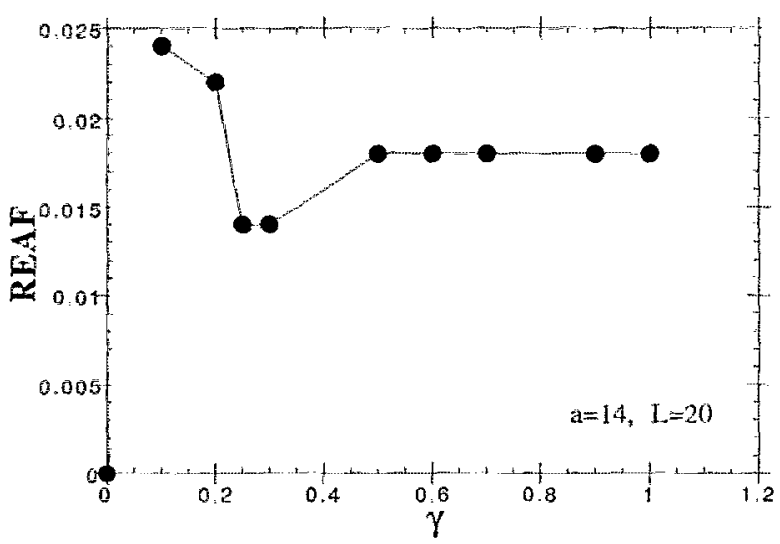

FIG. 5. Dependence of REAF on the parameter $\gamma$ in the system 2, for $a=14$ and $L=12$.

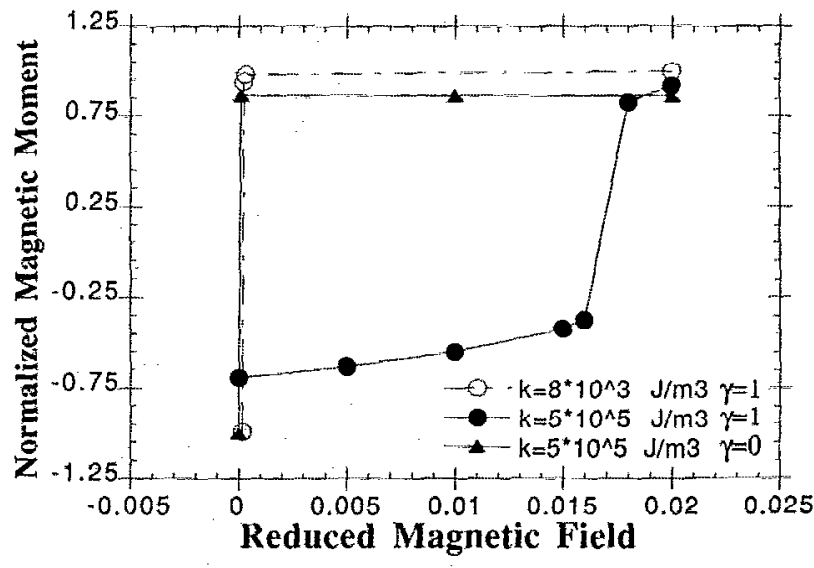

FIG. 6. Evolution with the reduced applied magnetic field $\left(\mu_{0} m H / 2 D\right)$ of the total magnetic moment, along the field direction and normalized to its saturation value, considering two different anisotropic inclusion: hard with $K=5 \times 10^{5} \mathrm{~J} \mathrm{~m}^{-3}$ and soft with $K=8 \times 10^{3} \mathrm{~J} \mathrm{~m}^{-3}$. The effect of exchange decoupled $(\gamma=0)$ hard inclusion was also plotted.

malized magnetic moment of the total system as a function of the reduced applied field for two types of precipitates: hard inclusion $\left(K=5 \times 10^{5} \mathrm{~J} \mathrm{~m}^{-3}\right)$ and soft inclusion ( $K$ $=8 \times 10^{3} \mathrm{~J} \mathrm{~m}^{-3}$ ). The switching field is about one hundred times larger if the precipitate is highly anisotropic and $\gamma=1$. If the hard inclusion does not interact by exchange coupling to the soft phase $\gamma=0$ no macroscopic hardening is obtained.

\section{CONCLUSIONS}

From the above discussion a basic conclusion can be extracted: Interphase exchange between the soft magnetic phase and the highly anisotropic $\mathrm{Fe}_{2} \mathrm{~B}$ precipitate increases in a very effective way the field necessary to reverse the global system magnetization. If both phases are exchange decoupled, nonmagnetic hardening is observed.

This exchange interaction can account for the increase of the effective anysotropy field in very soft Fe-rich nanocrystals after an adequate annealing at higher temperature.

'Y. Yoshizawa, S. Oguma, and K. Yamauchi, J. Appl. Phys. 64, 6044 (1988).

${ }^{2}$ R. Alben, J. J. Becker, and M. C. Chi, J. Appl. Phys. 49, 1653 (1978).

${ }^{3}$ G. Herzer, Mater. Sci. Eng. A-133, 1 (1990).

${ }^{4}$ E. Pulido, I. Navarro, and A. Hernado, IEEE Trans. Magn. 28, 2424 (1992).

${ }^{5}$ E. Callen, Y. J. Liu, and J. R. Cullen, Phys. Rev. B 16-1, 263 (1977).

${ }^{6}$ A. Hernando, J. Magn. Magn. Mater. 117, 154 (1992).

${ }^{7}$ A. Hernando, I. Navarro, and J. Gonzalez, Europhys. Lett. 20-2, 175 (1992). 
Journal of Applied Physics is copyrighted by the American Institute of Physics (AIP). Redistribution of journal material is subject to the AIP online journal license and/or AIP copyright. For more information, see http://ojps.aip.org/japo/japcr/jsp

Copyright of Journal of Applied Physics is the property of American Institute of Physics and its content may not be copied or emailed to multiple sites or posted to a listserv without the copyright holder's express written permission. However, users may print, download, or email articles for individual use. 
Journal of Applied Physics is copyrighted by the American Institute of Physics (AIP). Redistribution of journal material is subject to the AIP online journal license and/or AIP copyright. For more information, see http://ojps.aip.org/japo/japcr/jsp 